\title{
Endometriosis and Headache
}

\author{
Lars Jacob Stovner • Karen Agidius • Mattias Linde
}

Published online: 10 May 2011

(C) The Author(s) 2011. This article is published with open access at Springerlink.com

\begin{abstract}
Headache and endometriosis show some similarities in their clinical and epidemiological features that are probably due to the influence of female sexual hormones on both disorders. Epidemiological studies indicate that they are comorbid disorders. However, the nature of the comorbidity is not known with certainty, but a likely explanation may be common susceptibility genes. Another possibility is that, because they both are related to pain, increased pain sensitivity induced by one of the disorders may lead to a higher likelihood of developing the other, possibly mediated by nitrogen oxide or prostaglandins. A common link to the widespread use of estroprogestins may seem less probable. For physicians dealing with women with either of these disorders, awareness of the comorbidity may be helpful in the treatment of the patient.
\end{abstract}

Keywords Comorbidity · Endometriosis · Genes ·

Headache · Menarche $\cdot$ Migraine Perimenstrual Headache Fertility Prostaglandins $\cdot$ Nitric oxide

\section{Introduction}

From the point of view of headache science, the relation between headache and typical "women's" diseases like

\section{J. Stovner $(\bowtie) \cdot$ M. Linde}

Norwegian National Headache Centre, Department of

Neuroscience, Norwegian University of Science and Technology,

Trondheim University Hospital,

7006 Trondheim, Norway

e-mail: lars.stovner@ntnu.no

\section{K. Ægidius}

Department of Neurology, University Hospital of Copenhagen, Bispebjerg Hospital,

2400 Copenhagen, Denmark endometriosis is interesting because many features of headache, and migraine in particular, suggest a relation to female hormones. From a clinical perspective, it is important to know whether disorders are comorbid, and if so, to investigate the mechanisms for the comorbidity because this can have relevance for the choice of investigations to make and throw light on the pathophysiology and the etiology of both of them.

Endometriosis is a common health problem for women. Its exact prevalence is unknown because surgery is required for its diagnosis, but it is estimated to be present in 3\% to $10 \%$ of women of reproductive age and $25 \%$ to $35 \%$ of infertile women. It often is accompanied by chronic pelvic pain, which occurs in $15 \%$ to $24 \%$ of fertile women, and active endometriosis is found in about $33 \%$ of women with chronic pelvic pain [1]. The prevailing theory for the pathogenesis of endometriosis (Sampson's theory) posits that viable endometrium can flow retrogradely through the uterine tubes into the peritoneum during menstruation. Accordant with this theory, it has been found that women with increased exposure to menses (early menarche, short cycle length, long menstrual bleeding, and low parity) are at risk. However, these findings are not consistent, and it has been objected that some of them may also be the results, rather than the cause, of endometriosis [2•]. A particular problem for this "auto-transplant theory" is that it fails to explain endometriosis in women who have never menstruated [3]. Another hypothesis, also compatible with extraperitoneal cysts (eg, in the lungs), is that endometriosis is caused by small defects of embryogenesis [4].

Among the headaches, it is particularly for migraine that a relation to sex hormones has been studied. Migraine is especially common among fertile women, often starting around the age of menarche and improving after menopause [5]. About half of all women with migraine report an 
association with menstruation (menstrually related headache), though attacks exclusively in relation to menstruation (pure menstrual migraine) occur in only $5 \%$ to $8 \%$ of women with migraine $[6,7]$. Menstrual attacks seem to be precipitated by the abrupt fall of estrogen at the end of the luteal phase of the menstrual cycle. Although many factors may precipitate attacks, migraine now is mostly understood as a disorder of the brain, but vascular factors probably also play a role in the pathogenesis.

\section{Similar Features of Endometriosis and Headache}

Although migraine and endometriosis clearly affect different parts of the body, they share some clinical and epidemiological features, most of which probably can be accounted for by the influence of female sex hormones on both disorders.

Early menarche seems to be a risk factor for both headache $[8 \bullet]$ and endometriosis $[2 \bullet, 9 \bullet]$. For headache, it is of interest that age of menarche seems to influence the prevalence of headache, not only among adolescents but throughout life [8•]. Both psychological factors related to early physical maturation, as well as physiological factors related to higher estrogen levels or to increased estrogen sensitivity, have been invoked to explain why headache is more prevalent in females with early menarche. In the case of endometriosis, if the theory of retrograde flow of menstrual blood is true, all conditions (including early menarche) that lead to more menstruations will give a higher prevalence of endometriosis.

The fact that both migraine and endometriosis are related to early menarche could indicate that one disorder causes the other, but more likely, early menarche is a factor that increases the risk of both. Because menstruation is almost a prerequisite for developing endometriosis and because the hormonal fluctuation related to menstruation is a strong trigger for migraine attacks in women, it would seem logical that the earlier menstruation starts, the more likely it is that one or both disorders will develop. The relation of both disorders to early menarche also could explain the finding that migraine seems to start earlier in women with endometriosis [10].

The common relation to sex hormones probably explains similarities in relation to treatment with gonadotropinreleasing hormone (GnRH) agonists (GnRH-a). A Cochrane report has assessed GnRH-a to be effective against pain associated with endometriosis [11॰]. A case study has shown that migraine attacks abated during treatment with a GnRH-a for endometriosis [12]. In a small $(n=5)$, noncontrolled, open study, treatment with GnRH-a tended to relieve menstrual headaches also in women without endometriosis [13]. In another study on migrai- neurs without endometriosis, in which medical menopause was first induced with a GnRH-a and the women then received either estrogen add-back or placebo in a blinded fashion, it was found that the GnRH-a with estrogen was superior to the GnRH-a with placebo on all headaches [14]. The estrogen levels were probably stable in all patients, but the effect was most marked in the estrogen add-back group. It may be objected that in this study, the effect may be caused by the estrogen as much as by the GnRH-a.

It also should be noted that headache is commonly listed as a side effect of GnRH-a for treatment of endometriosis, but there seems to be less headache with this treatment than with danazol [11•]. In general, when headache is reported as a side effect, one often may question whether it is caused by the drug or whether it occurs incidentally during the drug treatment.

\section{Comorbidity}

Several studies indicate a comorbidity between headache and endometriosis (ie, that they co-occur in the same individual more often than what would be expected from chance). The first study to suggest this was published in 1975. Before an operation for pelvic pain, 125 women were asked about headache in the menstrual period. Those who, during operation, proved to have endometriosis externa (ie, outside the uterine cavity) had significantly more headache than participants with pain from other causes, and headache was almost as common as pelvic pain [15].

This relation has been confirmed in later studies. In a case-control study, endometriosis was more common in migraineurs than in the control patients without headache (22 vs $9.6 \%, P<0.01$ ). Among the women with migraine, headache on 15 days per month or more occurred significantly more often among those with than among those without comorbid endometriosis, and headacherelated disability also was significantly higher in migraineurs with endometriosis [16]. The group with migraine and endometriosis had more comorbid conditions, not only those that may be directly caused by endometriosis (eg, dysmenorrhea, metrorrhagia, and infertility), but they also had more depression, anxiety, irritable bowel syndrome, fibromyalgia, chronic fatigue syndrome, and interstitial cystitis. Migraineurs more often had symptoms of premenstrual dysphoric disorder than control patients, but there was no significant difference between the migraineurs with or without endometriosis. This is in accordance with the results from a small treatment study [17] showing a significant positive correlation between the severity of headache and premenstrual syndrome (PMS), and the data indicated that PMS symptoms were worsened by the presence of headache. 
Another case-control study [10] demonstrated a higher prevalence of headache among 133 women with endometriosis than among 166 control patients (63.9 vs $36.1 \%, P<$ $0.001)$. This difference only concerned migraine ( 38.3 vs $15.2 \%, P<0.01)$, being most marked for migraine with aura $(13.5 \%$ vs $1.2 \%, P<0.001)$. Virtually no difference was found with regard to tension-type headache (21 vs $22 \%$ ) [10].

\section{Mechanism for the Comorbidity}

An epidemiological association between migraine and endometriosis may represent methodological artifacts, but there also are some potential natural and iatrogenic mechanisms that could explain the association, which will be consider here.

One possibility is that endometriosis may cause migraine, or vice versa. Looking at the first option, it has been demonstrated that the pain in endometriosis is related to endometrial implants in pelvic tissue [18]. However, many studies have failed to demonstrate a consistent relation between the pain and the anatomy and biochemistry of the implants. In a rat model of endometriosis, it has been shown that the ectopic implants develop both sensory and autonomic nerve supply, mediating the nerve impulses through splanchnic nerves and the vagal nerve to the central nervous system. It is suggested that the implants may lead to central sensitization, causing an increased reaction to afferent impulses also from healthy organs, which can explain the relative lack of association between the localization of implants and the pain. Conceivably, this also may apply to sensory input from the head, decreasing the threshold for developing head pain. This mechanism would not be specific for endometriosis, which would be in accordance with the findings of a prospective study of 108 women undergoing laparoscopy for pelvic pain that had lasted at least 6 months. The prevalence of migraine was not higher among women with endometriosis than among those with other pelvic pain [19••]. However, this theory may not be corroborated by the finding that among patients with endometriosis and headache, no difference was found with regard to headache duration or frequency among patients with mild endometriosis and patients with severe endometriosis [10].

If increased sensitivity constitutes the link between endometriosis and headache, this effect could be mediated by prostaglandins or nitric oxide (NO). Prostaglandins are thought to play a role in pain transmission and migraine headache by decreasing noradrenergic transmission, sensitizing nociceptors, and promoting neurogenic inflammation $[20,21]$. Endometriosis is associated with a significant increase in prostaglandin production [22-24]. NO is an important molecule in the regulation of cerebral blood flow
[25•], but it also is involved in angiogenesis and nociceptive processing, and several lines of evidence support its role in both primary headaches [25] and endometriosis [26•, 27]. However, no study has to date shown that there is a direct link between prostaglandins or NO produced by the endometrial implants and migraine attacks, so this would at present seem highly speculative.

The reverse possibility, that migraine may somehow cause endometriosis or at least make its detection more likely, also should be considered. Migraineurs have been shown to have a lower threshold for pain not only during attacks and in the pre-attack phase [28•], but also in the interictal phase [29•]. This could mean that those with headache will suffer from more pain if they develop endometriosis, and thus, increase the likelihood that endometriosis is diagnosed. However, the fact that the presence and severity of migraine does not seem to influence the intensity of dysmenorrhea experienced by the women with endometriosis is not in accord with this theory [10].

Interestingly, it also has been hypothesized that migraine may lead to endometriosis in a more direct way. This is based on a study of 50 female migraineurs and 52 age- and sex-matched control patients [30]. Surgically confirmed endometriosis occurred more often in the migraine group ( $30 \%$ vs $4 \%, P<0.001)$. In addition, the migraine group had more often a history of self-reported menorrhagia ( $63 \%$ vs $37 \%$ ), bruising, rectal bleeding, and excessive bleeding after minor trauma $(P<0.01)$, even after correcting for use of oral contraceptives (OCs), hormone-replacement therapy (HRT), and NSAIDs. It seems likely that menorrhagia and a slight bleeding diathesis could result in an increased risk of retrograde menstruation, which, according to Sampson's theory, could induce endometriosis. However, a weakness of the study is that many patients in the migraine group had endometriosis, making it likely that the menorrhagia can be caused by the endometriosis, at least in some cases.

Also, the comorbidity may be explained by common causal factors. One such factor could be the use of estroprogestins because both headache and endometriosis show a similar, although not identical, relation to use of these medicines. For endometriosis, it is well established that OCs, for instance, represent a valuable treatment option for the disease by relieving pain and possibly preventing the disorder $[31 \bullet, 32 \cdot$. On the other hand, a recent metaanalysis of 18 studies showed that there is an increased risk of endometriosis in present and former users of OCs [33•]. It also has been demonstrated that the use of OCs and HRT are associated with headache [34, 35]. Therefore, one could hypothesize that the comorbidity may be due to widespread use of these substances. However, in spite of the positive epidemiological association between OC use and endometriosis, there may not be a direct causal link. In one study, 
women with and without endometriosis were asked about the reason they started with OCs. Women with endometriosis more often started it due to dysmenorrhoea, which was taken as an indication that early symptoms of endometriosis were the cause of the OC use, rather than the opposite $[31 \bullet]$. Similarly for headache, it has been discussed whether the positive association between headache and OC use or HRT is explained by the fact that many women use estrogen supplements to relieve headache. Therefore, OC use as cause of the comorbidity may be less plausible.

\section{Genetics}

An Australian study was performed in a cohort of 931 families with at least two sisters with endometriosis, using 815 independent monozygotic and 457 dizygotic female twin pairs as control patients [36••]. A significant additive genetic correlation was found, indicating common genetic influences to explain their co-occurrence within individuals. Analyses to explore the direction of the causation indicated an underlying genetic disposition common to both disorders. There was no evidence that neuroticism could explain the association. Further research is needed to detect the exact genetic risk loci.

Some genetic studies support a role of the estrogen receptor 1 gene in migraine susceptibility, although the exact variant is not identified [37, 38]. This receptor also has been reported to be associated with endometriosis $[39,40]$.

\section{Conclusions}

It seems likely that headache and endometriosis are comorbid disorders. The mechanism for the comorbidity is not known. It could be a common pathogenetic factor, such as common susceptibility genes, or it could be related to the fact that early menarche is associated with both disorders. Also, increased pain sensitivity induced by one of the disorders could make a diagnosis of the other more likely. Some observations indicate that increased sensitivity can be related to similarities in NO or prostaglandin production. Because both are very prevalent disorders, it may be important for the clinician dealing with one of the disorders to take the other into consideration to give the optimal treatment.

Disclosures Dr. Lars J. Stovner has served as a partner in a European Union research project (receiving a European Research Council grant); has received a research grant from AstraZeneca; and has received travel expense compensation from Pfizer. Dr. K. Aegidius: none. Dr. Mattias Linde has served on a chronic migraine advisory board for Allergan.
Open Access This article is distributed under the terms of the Creative Commons Attribution Noncommercial License which permits any noncommercial use, distribution, and reproduction in any medium, provided the original author(s) and source are credited.

\section{References}

Papers of particular interest, published recently, have been highlighted as:

- Of importance

-. Of major importance

1. Katz VL. Endometriosis. In: Katz VL, editor. Comprehensive gynecology. 5th ed. Philadelphia: Mosby Elsevier; 2007. MD Consult.

2. - Treloar SA, Bell TA, Nagle CM, Purdie DM, Green AC. Early menstrual characteristics associated with subsequent diagnosis of endometriosis. Am J Obstet Gynecol. 2010;202(6):534 e531-536. This is a case-control study showing that endometriosis is more common in women with early menarche.

3. Redwine DB. Was Sampson wrong? Fertil Steril. 2002;78(4):686-93.

4. Signorile PG, Baldi A. Endometriosis: new concepts in the pathogenesis. Int J Biochem Cell Biol. 2010;42(6):778-80.

5. Bille B. A 40-year follow-up of school children with migraine. Cephalalgia. 1997;17(4):488-91.

6. MacGregor EA. "Menstrual" migraine: towards a definition. Cephalalgia. 1996;16(1):11-21.

7. Couturier EG, Bomhof MA, Neven AK, van Duijn NP. Menstrual migraine in a representative Dutch population sample: prevalence, disability and treatment. Cephalalgia. 2003;23(4):302-8.

8. - Aegidius KL, Zwart JA, Hagen K, Dyb G, Holmen TL, Stovner LJ. Increased headache prevalence in female adolescents and adult women with early menarche. The Head-HUNT Studies. Eur J Neurol. 2011;18(2):321-8. This large population-based health survey showed that migraine and other headaches are more prevalent among women with early menarche.

9. Matalliotakis IM, Cakmak H, Fragouli YG, Goumenou AG, Mahutte NG, Arici A. Epidemiological characteristics in women with and without endometriosis in the Yale series. Arch Gynecol Obstet. 2008;277(5):389-393. This is another case-control study showing a relation between early menarche and endometriosis.

10. Ferrero S, Pretta S, Bertoldi S, Anserini P, Remorgida V, Del Sette $\mathrm{M}$, et al. Increased frequency of migraine among women with endometriosis. Hum Reprod. 2004;19(12):2927-32.

11. - Brown J, Pan A, Hart RJ. Gonadotrophin-releasing hormone analogues for pain associated with endometriosis. Cochrane Database Syst Rev. 2010;12:CD008475. This is a thorough evaluation of GnRH analogues for endometriosis. For the present review, it is relevant mostly for documenting headache as a side effect of the treatment.

12. Maggioni F, Palmieri A, Tropea M, Zanchin G. Influence of physiologic hormonal modification and of hormonal treatment in a patient with a history of migraine with aura. J Headache Pain. 2008;9(2):129-31.

13. Murray SC, Muse KN. Effective treatment of severe menstrual migraine headaches with gonadotropin-releasing hormone agonist and "add-back" therapy. Fertil Steril. 1997;67(2):390-3.

14. Martin V, Wernke S, Mandell K, Zoma W, Bean J, Pinney S, et al. Medical oophorectomy with and without estrogen add-back therapy in the prevention of migraine headache. Headache. 2003;43(4):309-21.

15. Tervila L, Marttila P. Headache as a symptom of endometriosis externa. Ann Chir Gynaecol Fenn. 1975;64(4):239-41. 
16. Tietjen GE, Bushnell CD, Herial NA, Utley C, White L, Hafeez F. Endometriosis is associated with prevalence of comorbid conditions in migraine. Headache. 2007;47(7):1069-78.

17. Martin VT, Wernke S, Mandell K, Ramadan N, Kao L, Bean J, et al. Symptoms of premenstrual syndrome and their association with migraine headache. Headache. 2006;46(1):125-37.

18. Berkley KJ, Rapkin AJ, Papka RE. The pains of endometriosis. Science (New York, NY). 2005;308(5728):1587-9.

19. •- Karp BI, Sinaii N, Nieman LK, Silberstein SD, Stratton P. Migraine in women with chronic pelvic pain with and without endometriosis. Fertil Steril. 2010. This study of women undergoing laparoscopy for pelvic pain shows a very high lifetime prevalence of migraine (67\%) within the group, but it was not more common among those with endometriosis compared to those with pelvic pain from other causes.

20. Kornstein SG, Parker AJ. Menstrual migraines: etiology, treatment, and relationship to premenstrual syndrome. Curr Opin Obstet Gynecol. 1997;9(3):154-9.

21. Sarchielli P, Alberti A, Codini M, Floridi A, Gallai V. Nitric oxide metabolites, prostaglandins and trigeminal vasoactive peptides in internal jugular vein blood during spontaneous migraine attacks. Cephalalgia. 2000;20(10):907-18.

22. De Leon FD, Vijayakumar R, Rao CV, Yussman M. Prostaglandin F2 alpha and E2 release by peritoneum with and without endometriosis. Int J Fertil. 1988;33(1):48-51.

23. Karck U, Reister F, Schafer W, Zahradnik HP, Breckwoldt M. PGE2 and PGF2 alpha release by human peritoneal macrophages in endometriosis. Prostaglandins. 1996;51(1):49-60.

24. Schenken RS, Asch RH. Surgical induction of endometriosis in the rabbit: effects on fertility and concentrations of peritoneal fluid prostaglandins. Fertil Steril. 1980;34(6):581-7.

25. - Olesen $\mathrm{J}$. The role of nitric oxide (NO) in migraine, tension-type headache and cluster headache. Pharmacol Ther. 2008;120 (2):157-171. This is a thorough review of the effects of NO and its potential roles in headache pathophysiology.

26. • Kim H, Ku SY, Kim SH, Lee GH, Choi YM, Kim JM, Lee TH, Moon SY. Endothelial nitric oxide synthase gene Glu298Asp polymorphism is associated with advanced stage endometriosis. Hum Reprod. 2009;24(10):2656-2659. In the present context, this report is interesting mainly because it shows that NO also can be of importance for endometriosis.

27. Wu MY, Chao KH, Yang JH, Lee TH, Yang YS, Ho HN. Nitric oxide synthesis is increased in the endometrial tissue of women with endometriosis. Hum Reprod. 2003;18(12):2668-71.

28. - Sand T, Zhitniy N, Nilsen KB, Helde G, Hagen K, Stovner LJ. Thermal pain thresholds are decreased in the migraine preattack phase. Eur J Neurol. 2008;15(11):1199-1205. This is a thorough study demonstrating lowered pain thresholds during the preattack phase of migraine.

29. - Moulton EA, Burstein R, Tully S, Hargreaves R, Becerra L, Borsook D. Interictal dysfunction of a brainstem descending modulatory center in migraine patients. PLoS One. 2008;3(11):e3799. This is an intriguing study demonstrating that central pain modulatory systems also may be altered between attacks of migraine.

30. Tietjen GE, Conway A, Utley C, Gunning WT, Herial NA. Migraine is associated with menorrhagia and endometriosis. Headache. 2006;46(3):422-8.

31. - Somigliana E, Vercellini P, Vigano P, Abbiati A, Benaglia L, Fedele L. Endometriosis and estroprogestins: the chicken or the egg causality dilemma. Fertil Steril. 2011;95(1):431-433. This study shows that although the use of OCs seems to be associated with endometriosis, the cause of this association may be that women use OCs to alleviate abdominal symptoms in the early phase of the disease.

32. - Practice Committee of American Society for Reproductive Medicine. Treatment of pelvic pain associated with endometriosis. Fertil Steril. 2008;90(5 Suppl):S260-269. The American Society for Reproductive Medicine has issued this thorough evaluation with practical treatment guidelines that also may be of help for physicians treating headache patients.

33. - Vercellini P, Eskenazi B, Consonni D, Somigliana E, Parazzini F, Abbiati A, et al. Oral contraceptives and risk of endometriosis: a systematic review and meta-analysis. Hum Reprod Update. 2010. This is a critical appraisal of the position of OCs in the treatment of endometriosis.

34. Aegidius K, Zwart JA, Hagen K, Schei B, Stovner LJ. Oral contraceptives and increased headache prevalence: the HeadHUNT Study. Neurology. 2006;66(3):349-53.

35. Aegidius KL, Zwart JA, Hagen K, Schei B, Stovner LJ. Hormone replacement therapy and headache prevalence in postmenopausal women. The Head-HUNT study. Eur J Neurol. 2007;14(1):73-8.

36. • Nyholt DR, Gillespie NG, Merikangas KR, Treloar SA, Martin NG, Montgomery GW. Common genetic influences underlie comorbidity of migraine and endometriosis. Genet Epidemiol. 2009;33(2):105-113. This is a large twin study confirming the comorbidity between the two disorders, and showing that this is to a large extent caused by shared genes.

37. Colson NJ, Lea RA, Quinlan S, Griffiths LR. No role for estrogen receptor 1 gene intron 1 Pvu II and exon 4 C325G polymorphisms in migraine susceptibility. BMC Med Genet. 2006;7:12.

38. Kaunisto MA, Kallela M, Hamalainen E, Kilpikari R, Havanka H, Harno $\mathrm{H}$, et al. Testing of variants of the MTHFR and ESR1 genes in 1798 Finnish individuals fails to confirm the association with migraine with aura. Cephalalgia. 2006;26(12):1462-72.

39. Kim SH, Choi YM, Jun JK, Kim JG, Moon SY. Estrogen receptor dinucleotide repeat polymorphism is associated with minimal or mild endometriosis. Fertil Steril. 2005;84(3):774-7.

40. Georgiou I, Syrrou M, Bouba I, Dalkalitsis N, Paschopoulos M, Navrozoglou I, et al. Association of estrogen receptor gene polymorphisms with endometriosis. Fertil Steril. 1999;72 (1):164-6. 